\title{
Retrospective comparison of focused shockwave therapy and radial wave therapy for men with erectile dysfunction
}

\author{
Shannon S. Wu ${ }^{1 \wedge}$, Kyle J. Ericson ${ }^{2}$, Daniel A. Shoskes ${ }^{2}$ \\ ${ }^{1}$ Cleveland Clinic Lerner College of Medicine, Cleveland, OH, USA; ${ }^{2}$ Department of Urology, Cleveland Clinic Foundation, Glickman Urological \\ and Kidney Institute, Cleveland, OH, USA \\ Contributions: (I) Conception and design: All authors; (II) Administrative support: DA Shoskes; (III) Provision of study materials or patients: DA \\ Shoskes, KJ Ericson; (IV) Collection and assembly of data: SS Wu, KJ Ericson; (V) Data analysis and interpretation: SS Wu, KJ Ericson; (VI) \\ Manuscript writing: All authors; (VII) Final approval of manuscript: All authors. \\ Correspondence to: Daniel A. Shoskes. 9500 Euclid Avenue, Desk Q10-1, Cleveland, OH, 44118, USA. Email: dshoskes@gmail.com.
}

Background: Low-intensity shockwave therapy (SWT) is an emerging treatment for erectile dysfunction (ED). Devices used for SWT include focused shockwave therapy (fSWT) or radial wave therapy (rWT), which differ in how the waves are generated, their tissue penetration, and the shape of their pressure waves. Most studies of SWT for ED to date have utilized fSWT. Although widely used, the efficacy of rWT for ED is unknown. Our objective is to compare the efficacy of rWT and fSWT for ED at our institution.

Methods: A retrospective chart review was performed to identify all men with ED treated by fSWT or rWT. Men with history suggesting non-vasculogenic ED were excluded. All men received 6 consecutive weekly treatments. The fSWT group received 3,000 shocks per treatment at $0.09 \mathrm{~mJ} / \mathrm{mm}^{2}$. The rWT group received 10,000 shocks per treatment at $90 \mathrm{~mJ}$ and $15 \mathrm{~Hz}$. Pre-treatment and 6-week post-treatment Sexual Health Inventory in Men (SHIM) scores were measured. Treatment response was categorized on a scale of 1-3 (1 if no improvement, 2 if erections sufficient for intercourse with phosphodiesterase 5 inhibitors (PDE5i), or 3 if sufficient erections without PDE5i). Primary endpoint was self-reported improvement score of 2 or greater.

Results: A total of 48 men were included: 24 treated by fSWT and 24 by rWT. There were no significant differences in age, duration of ED, pre-treatment PDE5i use, or pre-treatment SHIM scores between the groups. Following treatment with rWT, the mean SHIM score improved from 9.3 to $16.1(\mathrm{P}<0.001)$. The mean SHIM following fSWT improved from 9.3 to $15.5(\mathrm{P}<0.001)$. The mean improvement in SHIM score did not differ between rWT (6.8) and fSWT (6.2) (P=0.42). 54\% of men treated by fSWT experienced a significant clinical improvement ( $\geq$ grade 2 response) compared to $75 \%$ in the rWT group ( $\mathrm{P}=0.42)$. There were no reported side effects with either device.

Conclusions: In our patient population, both fSWT and rWT were moderately effective treatments for arteriogenic ED with no observable difference in efficacy between the two modalities.

Keywords: Low-intensity shockwave therapy (low-intensity SWT); radial shockwave therapy; focused shockwave therapy (fSWT); erectile dysfunction (ED)

Submitted May 04, 2020. Accepted for publication Sep 16, 2020.

doi: 10.21037/tau-20-911

View this article at: http://dx.doi.org/10.21037/tau-20-911

\footnotetext{
^ ORCID: 0000-0002-0809-9143.
} 


\section{Introduction}

Low intensity shockwave therapy (SWT) is an emerging treatment option for men with vasculogenic erectile dysfunction (ED). The efficacy of SWT in this setting has been evaluated in several randomized trials with varying benefit (1-7). While there is substantial heterogeneity in treatment regimens employed and the devices used, metaanalyses of these trials suggest men with vasculogenic ED experience a significant improvement in erectile function after SWT (8-11). To date, all pre-clinical and clinical trials thus far have utilized focused shockwave therapy (fSWT) (12). Radial wave therapy (rWT) is an alternative method of creating acoustic waves that is commonly utilized in orthopedics, physical therapy, and dermatology, but has not been evaluated for use in men with ED (13-16). The effect of rWT on men with vasculogenic ED, while often marketed as evidence-based ED treatment modality, is unknown.

Extracorporeal shockwaves used in medicine entail an acoustic wave of energy that travels through tissues and releases a rapid rise and fall of pressure at tissue interfaces, known as a shockwave. Acoustic waves can be delivered to tissues by two distinct mechanisms: focused shockwaves and non-focused radial waves. The two types of shockwaves differ substantially in their depth of tissue penetration, ability to focus the shockwave, and the rapidity of the rise and fall of pressure (shape of the shockwave). The different waveforms may produce varying biological effects, but these differences for specific indications remain largely unknown.

Focused shockwaves, which are the shockwaves used for extracorporeal shockwave lithotripsy treatment of urolithiasis, can be targeted to focal points at various tissue depths (up to $10-12 \mathrm{~cm}$ ) by utilizing reflection of energy created by an acoustic wave source such that the waves convene at a focus point for maximal energy, limiting energy dispersion and collateral damage to adjacent tissues (17). The energy profile of a focused shockwave entails a rapid (10 nanoseconds) rise and fall of the pressure wave. Focused shockwaves are generated by initiating a pressure wave via three distinct mechanisms: piezoelectric, electromagnetic, and electrohydraulic. These create a unique pressure wave that can be directed at a focal point (18). FSWT devices are currently FDA class II devices, which limits use to physicians typically in IRB-approved research protocols.

In contrast, the maximal point of energy of a radial wave, sometimes referred to as a dispersive shockwave, is at the tip of the device (19). These acoustic waves then disperse radially away from the tip of the device with rapid energy attenuation. The depth of penetration of radial waves varies based on energy input, but can reach up to $3.5 \mathrm{~cm}$ in human tissues. The energy profile entails a slower (5-10 microseconds) rise and fall of pressure than a focused shockwave. Radial waves are generated by a mechanical concussion in which a ballistic projectile repeatedly strikes an endplate and generates the dispersive acoustic wave. There are two mechanisms used to force the bullet against the endplate: pneumatic air compression and an electromagnetic system. rWT devices are currently FDA class I devices that do not require regulatory approval and may be used by anyone, with or without medical training.

At our clinic we have used both types of machines for clinical trials and also offer therapy outside of these trials based on a clinical diagnosis of vasculogenic ED. The objective of this study is to compare the effectiveness of $\mathrm{rWT}$ and $\mathrm{fSWT}$ on ED. We hypothesize that rWT is non-inferior to fSWT for treatment of ED. We present the following article in accordance with the STROBE reporting checklist (available at http://dx.doi. org/10.21037/tau-20-911).

\section{Methods}

We performed an IRB approved retrospective review of all men with ED that were treated with either fSWT or rWT outside of other existing SWT research protocols between 2017 and 2019 at our institution in two clinical locations. 48 patients included in the study were evaluated by a single urologist (DS). The use fSWT or rWT was dictated by clinic location where the treatment was administered; the fSWT machine was located at one outpatient clinic location and the rWT machine at another, neither the patients nor the urologist chose the SWT modality. Patients were excluded from analysis if they had ED of known neurologic cause (e.g., post radical prostatectomy), untreated hypogonadism, or a clinical diagnosis of psychogenic ED. rWT was performed using the Zimmer enPuls Pro (Zimmer MedizinSysteme GmbH, Neu-Ulm, Germany), which utilizes an electromagnetically produced radial pulse. fSWT was performed using the UroGold 100TM (Tissue Regeneration Technologies LLC, Woodstock, Georgia), which utilizes an electrohydraulic pulse generator. In both groups, patients underwent six consecutive weekly treatments using the settings recommended by the manufacturer. The fSWT regimen entailed 3,000 shocks per session at $0.09 \mathrm{~mJ} / \mathrm{mm}^{2}$. The $\mathrm{rWT}$ regimen entailed 
Table 1 Baseline characteristics of men that underwent focused shockwave therapy (fSWT) and radial wave therapy (rWT)

\begin{tabular}{lccc}
\hline Characteristic & $f S W T(n=24)$ & rWT $(n=24)$ & $P$ \\
\hline Age, median [IQR] & $61[48-70]$ & $61[54-69]$ & 0.90 \\
$\begin{array}{lccc}\text { Duration of ED in months, } \\
\text { median [IQR] }\end{array}$ & $61[36-61]$ & $68[12-96]$ & 0.67 \\
PDE5i use, n [\%] & $19[79]$ & $22[92]$ & 0.42 \\
Number of treatments, mean & 6.1 & 6.0 & 0.84 \\
$\begin{array}{l}\text { Pre-treatment SHIM, mean } \\
\text { [IQR] }\end{array}$ & $9.3[6-12]$ & $9.3[5-13]$ & 1.00 \\
\hline
\end{tabular}

IQR, interquartile range; ED, erectile dysfunction; PDE5i, phosphodiesterase-5 inhibitor; SHIM, Sexual Health Inventory in Men.

10,000 shocks per session at $15 \mathrm{~Hz}$ and $90 \mathrm{~mJ}$. The penis was manually, gently stretched for delivery of shockwaves to six treatment sites: one at each crus of the penis on the perineum and two locations on the shaft bilaterally. The study was conformed to the provisions of the Declaration of Helsinki (as revised in 2013). This study was reviewed and approved by the Cleveland Clinic Institutional Review Board (IRB No. 12-118). Informed consent was waived for this retrospective study as no direct interventions to the patient were present.

To assess the efficacy of treatment, pre-treatment and 6-week post-treatment Sexual Health Inventory in Men (SHIM) scores (20) were obtained by survey in clinic (20). At 6 weeks post-treatment, men were also asked whether their erections were sufficient for penetration and whether they required phosphodiesterase 5 inhibitors (PDE5i) to achieve an erection sufficient for penetration. Patients were instructed to try PDE5i if they did not have full erections after SWT. Those who did not already have a supply received a prescription from the study investigators. These responses were graded as follows: 1 if erections insufficient for penetration, 2 if erections were sufficient for penetration with the assistance of PDE5i, and 3 if erections were sufficient without requiring PDE5i. The secondary endpoint is a self-reported improvement of grade 2 or greater.

To assess the efficacy of rWT and fSWT we compared the pre- and post-treatment SHIM scores. The mean change in SHIM scores (delta SHIM) and the proportion of men achieving a grade 2 or better response between the two treatment groups (i.e., erections sufficient for penetration with or without PDE5i) were compared. Potential confounders include placebo effect due to nature of the intervention requiring a generator.

\section{Statistical analysis}

Intra-group comparisons of continuous data were made with a paired $t$-test, while an unpaired t-test was used for inter-group comparisons. Categorical data was compared with a chi-squared test. $\mathrm{P}$ values $<0.05$ were considered statistically significant. Statistical analyses were performed using SPSS version 23.0 (IBM, Armonk, New York, USA).

\section{Results}

Forty-eight men with ED who met our criteria were treated with SWT: 24 men with fSWT and 24 with rWT. The median age of patients was 65.6 (IQR, 51-70), median pre-treatment SHIM 8 (IQR, 5-13), and $85 \%(41 / 48)$ used PDE5i. Table 1 shows the baseline characteristics of each cohort. There was no statistical difference between the fSWT and rWT groups in age (61.3 vs. 60.5 years), duration of ED symptoms (61 vs. 72 months), pre-treatment use of PDE5i (79\% vs. 92\%), or the baseline SHIM score (9.3 \pm 4.2 vs. 9.3 \pm 4.8$)$. All patients completed their 6-week treatment course. All patients had pre- and post-treatment SHIM scores and no missing data or loss to follow-up requiring imputation.

At 6 weeks post-treatment, there was a clinically and statistically significant increase in SHIM scores with both modalities (Figure 1). The mean SHIM of patients treated by rWT improved from 9.3 to 16.1 (SD 5.9) $(\mathrm{P}<0.0001)$. Similarly, the mean SHIM of patients treated by fSWT improved from 9.3 to 15.5 (SD 5.7) $(\mathrm{P}<0.001)$. There was no statistical difference in the delta SHIM between $\mathrm{rWT}$ and fSWT (P=0.42; Figure 2).

Among men treated by rWT, 25\% (6/24) reported no improvement (grade 1), 42\% (10/24) reported erections sufficient for penetration with the assistance of PDE5i (grade 2), and 33\% (8/24) reported sufficient erections without needing PDE5i (grade 3). In the fSWT group, $46 \%(11 / 24)$ reported no improvement (grade 1), 17\% (4/24) reported erections sufficient for penetration with the assistance of PDE5i (grade 2), and 37.5\% (9/24) reported sufficient erections without needing PDE5i (grade 3). In the rWT group, $75 \%(18 / 24)$ had a $\geq$ grade 2 response compared to $54 \%(13 / 24)$ in the fSWT group; however, this was not a statistically significant difference $(\mathrm{P}=0.42)$.

None of the patients included in this study experienced 

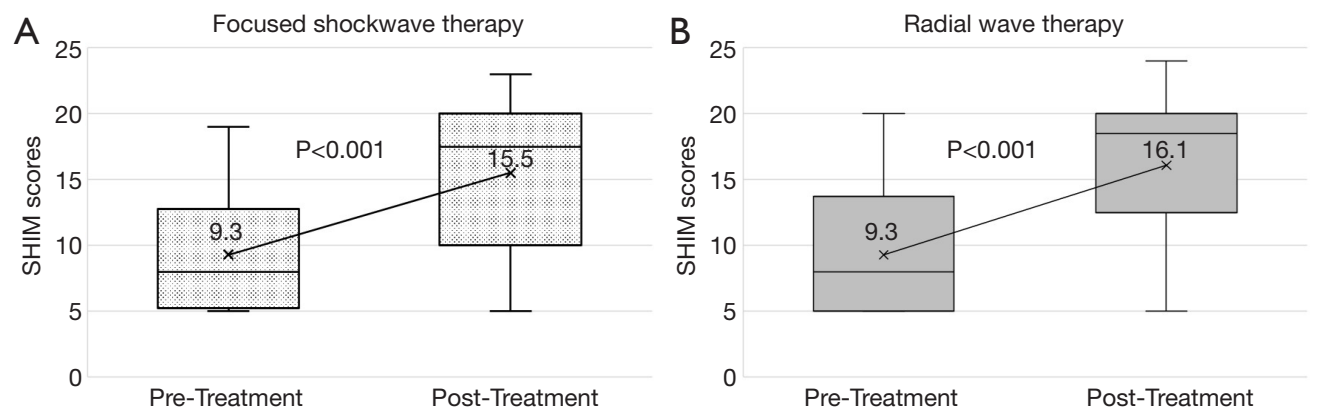

Figure 1 Box and whisker plots of pre- and post-treatment Sexual Health Inventory in Men (SHIM) scores for men treated by (A) focused shockwave therapy and (B) radial wave therapy (rWT). X-marks within the box plot denote the mean SHIM score.

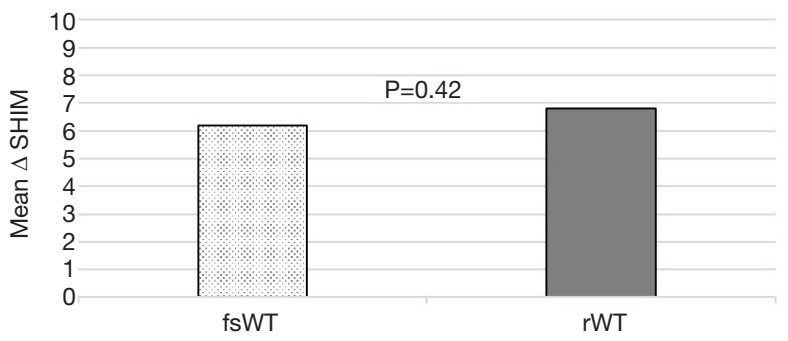

Figure 2 Mean change in Sexual Health Inventory in Men (SHIM) score from pre- to post-treatment with focused shockwave therapy (fSWT) and radial wave therapy (rWT).

an adverse event.

\section{Discussion}

Low-intensity SWT is emerging as a non-invasive alternative or supplemental option to PDE5 inhibitors for men with vasculogenic ED. To date, preclinical and clinical studies have utilized fSWT to deliver shockwaves. Despite the absence of clinical studies, rWT use is becoming widespread, which may be due to the ease of use of these devices and minimal FDA restrictions. $\mathrm{rWT}$ is widely used in other fields for indications such as venous ulcers and plantar fasciitis with high-quality evidence to support its use $(21,22)$. In this retrospective study, we demonstrated that rWT was clinically efficacious in treating men with a history suggestive of vasculogenic ED (mean SHIM improvement of 6.8), and clinical outcomes were not appreciably different between men treated by rWT and fSWT.

The proposed mechanism of action of SWT includes microtrauma that stimulates angiogenesis, stem cell proliferation, and nerve regeneration $(23,24)$. In rat models of ED, SWT lead to increased vascular endothelial growth factor
(VEGF) and endothelial nitric oxide synthase (eNOS) release within the corpus cavernosum (25). Because these studies were performed using focused shockwaves, the biological effects of rWT are less well-understood. Nevertheless, if the radial wave penetrates the tissue of interest and causes non-lethal trauma to the target cells, it is reasonable to assume that the injury response would be agnostic to the energy source of the wave, especially if an equivalent clinical response is seen. Side effects of both fSWT and rWT are minimal and shortterm, including bruising, swelling, paresthesia, or pain in the treatment area (26).

Prior SWT trials have shown efficacy with fSWT generators such as Direx MoreNova (27), Stortz Duolith SD1 (28,29), and Omnispec ED1000 (1,2,27-29). Several meta-analyses have shown focused shockwave therapies to be clinically favorable in ED (8-11). In a meta-analysis of seven randomized sham-controlled trials, Clavijo et al. reported a SHIM improvement of 6.4 points after fSWT compared to 1.65 in those treated with sham therapy (8). Several subsequent meta-analyses demonstrated that men treated with FSWT experienced SHIM improvements of 2.0-4.23 (9-11,26,30). Most studies included in these metaanalyses prohibited PDE5i use during fSWT treatment, highlighting the independent efficacy of fSWT therapy in patients who likely did not respond to first-line PDE5i treatment. This data suggests utility of fSWT for ED in PDE5i non-responders or for its dual use with PDE5i.

rWT has garnered increasing use and attention in nonurological fields. The orthopedic field has extensively used rWT for treatment of conditions such as biceps tendinopathy (31), knee tendinopathy (15), and plantar fasciitis $(15,31,32)$. A meta-analysis of fSWT and rWT in soft-tissue musculoskeletal injuries showed that the treatment modalities are equally safe, with varying efficacy 
for different injuries, although no direct head-to-head comparisons have been done (13).

This study has several limitations. The retrospective design, relatively small sample size, and unblinded nature of treatment preclude the ability to make definitive conclusions of efficacy of either modality against sham. Although $24 \%(6 / 24)$ and $46 \%(11 / 24)$ of patients reported no improvement in the rWT and fSWT groups, respectively, the small number of patients in each arm may have precluded the detection of a statistically significant difference. The findings from this pilot study are unable to provide definitive guidelines of superiority of one technique over another. However, larger studies powered for a superiority analysis may corroborate our findings more definitively. In keeping with most ED trials, a placebo effect likely accounts for a portion of the clinical benefit observed in both study arms. However, our data align with the clinical benefit observed in sham-controlled fSWT trials, suggesting the benefit is not due to placebo alone. The benefit seen in both the fSWT and rWT group in our study is strikingly similar. Long-term efficacy was not assessed as most patients were an out-of-town referral population. The usage of PDE5i is a potential confounding factor that limits the ability to draw conclusions about efficacy of SWT as a stand-alone treatment. However, 8/24 (33\%) and 9/24 $(37.5 \%)$ of men treated with rWT and fSWT, respectively, reported grade 3 improvement not requiring use of PDE5i after SWT, suggesting the independent efficacy of both SWT modalities in a sizable minority of men. Additionally, PDE5i use after SWT is commonly performed in clinical practice, and SWT may enhance the therapeutic effects of PDE5i in a synergistic manner (9).

In our patient population, low intensity SWT with either radial waves or focused shockwaves were clinically beneficial for men with a history suggestive of vasculogenic ED. Similar results were found between the two treatment modalities in our study; however, further studies with larger samples are needed to confirm our results before any recommendations on this topic are made. While limited by its retrospective nature, lack of randomization (although neither patient nor doctor had a choice of therapy) and lack of sham control, this suggests rWT is equally efficacious to fSWT in the treatment of vasculogenic ED and warrants further investigation in clinical trials.

\section{Acknowledgments}

Funding: None.

\section{Footnote}

Reporting Checklist: The authors have completed the STROBE reporting checklist. Available at http://dx.doi. org/10.21037/tau-20-911

Data Sharing Statement: Available at http://dx.doi. org/10.21037/tau-20-911

Peer Review File: Available at http://dx.doi.org/10.21037/ tau-20-911

Conflicts of Interest: All authors have completed the ICMJE uniform disclosure form (available at http://dx.doi. org/10.21037/tau-20-911). DAS serves as an unpaid editorial board member of Translational Andrology and Urology from Dec 2017 to Jul 2022. DAS reports investment from Triurol, paid consultant from UroGen, outside the submitted work. The other authors have no conflicts of interest to declare.

Etbical Statement: The authors are accountable for all aspects of the work in ensuring that questions related to the accuracy or integrity of any part of the work are appropriately investigated and resolved. The study was conformed to the provisions of the Declaration of Helsinki (as revised in 2013). This study was reviewed and approved by the Cleveland Clinic Institutional Review Board (IRB No. 12-118). Informed consent was waived for this retrospective study as no direct interventions to the patient were present.

Open Access Statement: This is an Open Access article distributed in accordance with the Creative Commons Attribution-NonCommercial-NoDerivs 4.0 International License (CC BY-NC-ND 4.0), which permits the noncommercial replication and distribution of the article with the strict proviso that no changes or edits are made and the original work is properly cited (including links to both the formal publication through the relevant DOI and the license). See: https://creativecommons.org/licenses/by-nc-nd/4.0/.

\section{References}

1. Vardi Y, Appel B, Kilchevsky A, et al. Does low intensity extracorporeal shock wave therapy have a physiological effect on erectile function? Short-term results of a randomized, double-blind, sham controlled study. J Urol 
2012;187:1769-75.

2. Yee CH, Chan ESY, Hou SSM, et al. Extracorporeal shockwave therapy in the treatment of erectile dysfunction: A prospective, randomized, double-blinded, placebo controlled study. Int J Urol 2014;21:1041-5.

3. Kalyvianakis D, Memmos E, Mykoniatis I, et al. LowIntensity Shockwave Therapy for Erectile Dysfunction: A Randomized Clinical Trial Comparing 2 Treatment Protocols and the Impact of Repeating Treatment. J Sex Med 2018;15:334-45.

4. Srini VS, Reddy RK, Shultz T, et al. Low intensity extracorporeal shockwave therapy for erectile dysfunction: A study in an Indian population. Can J Urol 2015;22:7614-22.

5. Costa P, Dias J, Gouveia R, et al. Low intensity extracorporeal shockwave therapy on erectile dysfunction-first results from a prospective study. AME Med J 2019;4:32.

6. Feldman R, Denes B, Appel B, et al. PD45-10 The safety and efficacy of LI-ESWT in 604 patients for erectile dysfunction: summary of current and evolving evidence. J Urol 2015;193:e905-6.

7. Kitrey ND, Gruenwald I, Appel B, et al. Penile Low Intensity Shock Wave Treatment is Able to Shift PDE5i Nonresponders to Responders: A Double-Blind, Sham Controlled Study. J Urol 2016;195:1550-5.

8. Clavijo RI, Kohn TP, Kohn JR, et al. Effects of LowIntensity Extracorporeal Shockwave Therapy on Erectile Dysfunction: A Systematic Review and Meta-Analysis. J Sex Med 2017;14:27-35.

9. Lu Z, Lin G, Reed-Maldonado A, et al. Low-intensity Extracorporeal Shock Wave Treatment Improves Erectile Function: A Systematic Review and Meta-analysis. Eur Urol 2017;71:223-33.

10. Dong L, Chang D, Zhang X, et al. Effect of Low-Intensity Extracorporeal Shock Wave on the Treatment of Erectile Dysfunction: A Systematic Review and Meta-Analysis. Am J Mens Health 2019;13:1557988319846749.

11. Campbell JD, Trock BJ, Oppenheim AR, et al. Metaanalysis of randomized controlled trials that assess the efficacy of low-intensity shockwave therapy for the treatment of erectile dysfunction. Ther Adv Urol 2019;11:1756287219838364.

12. Sokolakis I, Dimitriadis F, Teo P, et al. The Basic Science Behind Low-Intensity Extracorporeal Shockwave Therapy for Erectile Dysfunction: A Systematic Scoping Review of Pre-Clinical Studies. J Sex Med 2019;16:168-94.

13. Speed C. A systematic review of shockwave therapies in soft tissue conditions: Focusing on the evidence. Br J Sports Med 2014;48:1538-42.

14. van der Worp H, van den Akker-Scheek I, van Schie H, et al. ESWT for tendinopathy: technology and clinical implications. Knee Surg Sports Traumatol Arthrosc 2013;21:1451-8.

15. Liao CD, Xie GM, Tsauo JY, et al. Efficacy of extracorporeal shock wave therapy for knee tendinopathies and other soft tissue disorders: A meta-analysis of randomized controlled trials. BMC Musculoskelet Disord 2018;19:278.

16. Adatto MA, Adatto-Neilson RM. Facial treatment with acoustic wave therapy for improvement of facial skin texture, pores and wrinkles. J Cosmet Dermatol 2020;19:845-9.

17. Elmansy HE, Lingeman JE. Recent advances in lithotripsy technology and treatment strategies: A systematic review update. Int J Surg 2016;36:676-80.

18. Katz JE, Clavijo RI, Rizk P, et al. The Basic Physics of Waves, Soundwaves, and Shockwaves for Erectile Dysfunction. Sexual Medicine Reviews 2020;8:100-5.

19. Romeo P, Lavanga V, Pagani D, et al. Extracorporeal Shock Wave Therapy in Musculoskeletal Disorders: A Review. Med Princ Pract 2014;23:7-13.

20. Rosen RC, Cappelleri JC, Gendrano N. The International Index of Erectile Function (IIEF): a state-of-the-science review. Int J Impot Res 2002;14:226-44.

21. Dolibog P, Dolibog P, Franek A, et al. Randomized, controlled clinical pilot study of venous leg ulcers treated with using two types of shockwave therapy. Int J Med Sci 2018;15:1275-85.

22. Dedes V, Tzirogiannis K, Polikandrioti M, et al. Radial extra corporeal shockwave therapy versus ultrasound therapy in the treatment of plantar fasciitis. Acta Inform Med 2019;27:45-9.

23. Qiu X, Lin G, Xin Z, et al. Effects of low-energy shockwave therapy on the erectile function and tissue of a diabetic rat model. J Sex Med 2013;10:738-46.

24. Li H, Matheu MP, Sun F, et al. Low-energy Shock Wave Therapy Ameliorates Erectile Dysfunction in a Pelvic Neurovascular Injuries Rat Model. J Sex Med 2016;13:22-32.

25. Zhu GQ, Jeon SH, Bae WJ, et al. Efficient Promotion of Autophagy and Angiogenesis Using Mesenchymal Stem Cell Therapy Enhanced by the Low-Energy Shock Waves in the Treatment of Erectile Dysfunction. Stem Cells Int 2018;2018:1302672.

26. Roerdink RL, Dietvorst M, van der Zwaard B, et al. 
Complications of extracorporeal shockwave therapy in plantar fasciitis: Systematic review. Int J Surg 2017;46:133-45.

27. Patel P, Katz J, Lokeshwar SD, et al. Phase II Randomized, Clinical Trial Evaluating 2 Schedules of Low-Intensity Shockwave Therapy for the Treatment of Erectile Dysfunction. Sex Med 2020;8:214-22.

28. Tsai CC, Wang CJ, Lee YC, et al. Low-Intensity Extracorporeal Shockwave Therapy Can Improve Erectile Function in Patients Who Failed to Respond to Phosphodiesterase Type 5 Inhibitors. Am J Mens Health 2017;11:1781-90.

29. Chung E, Cartmill R. Evaluation of clinical efficacy, safety and patient satisfaction rate after low-intensity extracorporeal shockwave therapy for the treatment of male erectile dysfunction: an Australian first open-

Cite this article as: Wu SS, Ericson KJ, Shoskes DA. Retrospective comparison of focused shockwave therapy and radial wave therapy for men with erectile dysfunction. Transl Androl Urol 2020;9(5):2122-2128. doi:10.21037/tau-20-911 label single-arm prospective clinical trial. BJU Int 2015;115:46-9.

30. Baccaglini W, Pazeto CL, Corrêa Barros EA, et al. The Role of the Low-Intensity Extracorporeal Shockwave Therapy on Penile Rehabilitation After Radical Prostatectomy: A Randomized Clinical Trial. J Sex Med 2020;17:688-94.

31. Furia JP, Rompe JD, Maffulli N, et al Radial Extracorporeal Shock Wave Therapy Is Effective and Safe in Chronic Distal Biceps Tendinopathy. Clin J Sport Med 2017;27:430-7.

32. Wang YC, Huang H, Cheng S. Efficacy of Different Energy Levels Used in Focused and Radial Extracorporeal Shockwave Therapy in the Treatment of Plantar Fasciitis: A Meta-Analysis of Randomized Placebo-Controlled Trials. J Clin Med 2019;8:1497. 